\title{
A Utilização de Filtros Gaussianos na Análise de Imagens Digitais
}

\author{
Edison O. Jesus, $\quad$ Roberto Costa Jr. \\ Universidade Federal de Itajubá, UNIFEI \\ Instituto de Matemática e Computação, IMC \\ 37500-903, Itajubá, MG \\ e-mail: edison@unifei.edu.br \\ rcosta62br@unifei.edu.br
}

Resumo: Este trabalho tem por objetivo mostrar a utilização dos filtros Gaussianos no processamento digital de imagens, através de um aplicativo computacional especialmente desenvolvido para este fim. Com a utilização do aplicativo, pode-se alem de se obter um aprendizado matemático sobre curvas, obter conhecimentos sobre convolução de imagens, implementar vários tipos de filtros e entender seus efeitos sobre as imagens, entender os vários tipos de ruídos em imagens e com isto, melhorar a utilização de técnicas utilizadas em restauração de imagens. Enfim, é um aplicativo com utilização na matemática, na computação gráfica e também no processamento digital de imagens.

\section{A Curva de Gauss}

A distribuição Normal, também conhecida como curva de Gauss ou gaussiana é uma das mais importantes curvas utilizadas na matemática e na estatística.

Segundo [5], a distribuição gaussiana foi primeiramente introduzida pelo matemático Abraham de Moivre em um artigo no ano 1733, que foi reproduzido na segunda edição de The Doctrine of Chances (1738) no contexto da aproximação de distribuições binomiais para grandes valores de $n$. Seu resultado foi estendido por Laplace, em seu livro Analytical Theory of Probabilities (1812), e a partir daí é chamado o teorema de Moivre-Laplace.

Laplace usou a distribuição normal na análise de erros de experimentos, o importante método dos mínimos quadrados que foi introduzido por Legendre, em 1805. O termo distribuição normal foi inventado independentemente por Charles S. Peirce, Francis Galton e Wilhelm Lexis, por volta de 1875.

A curva de Gauss é descrita por seus parâmetros de média e desvio padrão, ou seja, conhecendo-se estes parâmetros consegue-se determinar qualquer probabilidade em uma Distribuição Normal, a qual pode ser utilizada na aproximação para o cálculo de outras distribuições quando o número de observações é grande [4].

A curva de Gauss de um conjunto $X$ é definida pela equação 1:

$$
G(x)=\frac{1}{\sigma \sqrt{2 \pi}} e^{-\frac{(\mu-x)^{2}}{2 \sigma^{2}}}
$$

Onde:

\begin{tabular}{|l|l|}
\hline$x$ & Conjunto com $n$ valores, tal que $-\infty<\mathrm{x}<\infty$ \\
\hline$G$ & Distribuição gaussiana dos valores de $X$ \\
\hline$\sigma$ & Desvio padrão dos valores de $X$, tal que $\sigma>0$ \\
\hline$\mu$ & Média dos valores de $X$ \\
\hline
\end{tabular}

Esta distribuição gaussiana tem o formato mostrado na figura 1, extraída de [5].

Se a variável aleatória $X$ tem como parâmetros $\mu=0$ e $\sigma=1$, a distribuição é chamada de distribuição normal padrão e a função de Gauss reduz-se à equação 2. Neste caso, o valor máximo de $G(x)$ é igual a 0.399 . 


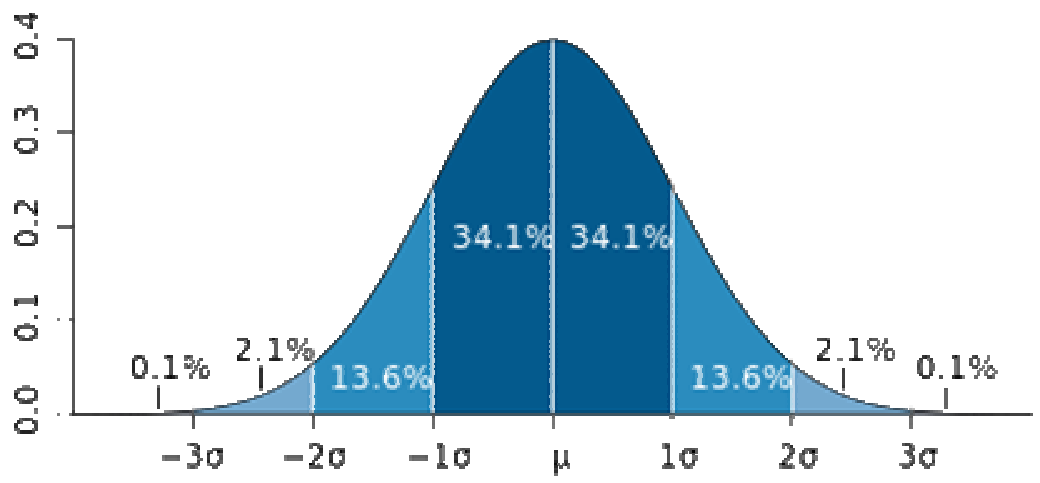

Figura 1 - forma da curva de distribuição gaussiana

$$
G(x)=\frac{1}{\sqrt{2 \pi}} e^{-\frac{x^{2}}{2}}
$$

Como exemplo, seja um conjunto $\mathrm{X}$ contendo os valores mostrados na tabela 1:

$\{1,4,7,4,1,4,9,9,8,4,7,8,5,8,7,4,7,8,7,4,4,1,4,7,4,1\}$

Tabela 1 - conjunto $X$

A média $\mu$ do conjunto $\mathrm{X}$ é calculada segundo a equação 3:

$$
\mu=\frac{1}{n} \sum_{k=1}^{n} x_{k}
$$

Para o exemplo da tabela 1, a média calculada para X é igual a 5.27.

A variância $\delta$ do conjunto X é calculada segundo a equação 4:

$$
\delta=\frac{1}{n-1} \sum_{k=1}^{n}(\mu-x)^{2} \quad \text { (eq. } 4 \text { ) }
$$

O desvio padrão $\sigma$ do conjunto $\mathrm{X}$ é calculado segundo a equação 5:

$$
\sigma=\sqrt{\delta}
$$

Para o exemplo da tabela 1, a variância calculada para $\mathrm{X}$ é igual a 6.50, e, portanto, $\mathrm{o}$ desvio padrão é igual a $\sqrt{6.50}=2.55$.

A figura 2 mostra os resultados obtidos para o cálculo do conjunto $\mathrm{X}$, bem como a sua média e seu desvio padrão.

Este resultado foi obtido através de um aplicativo computacional especialmente desenvolvido para este fim.

Figura 2 - curva de Gauss dos elementos da tabela 1

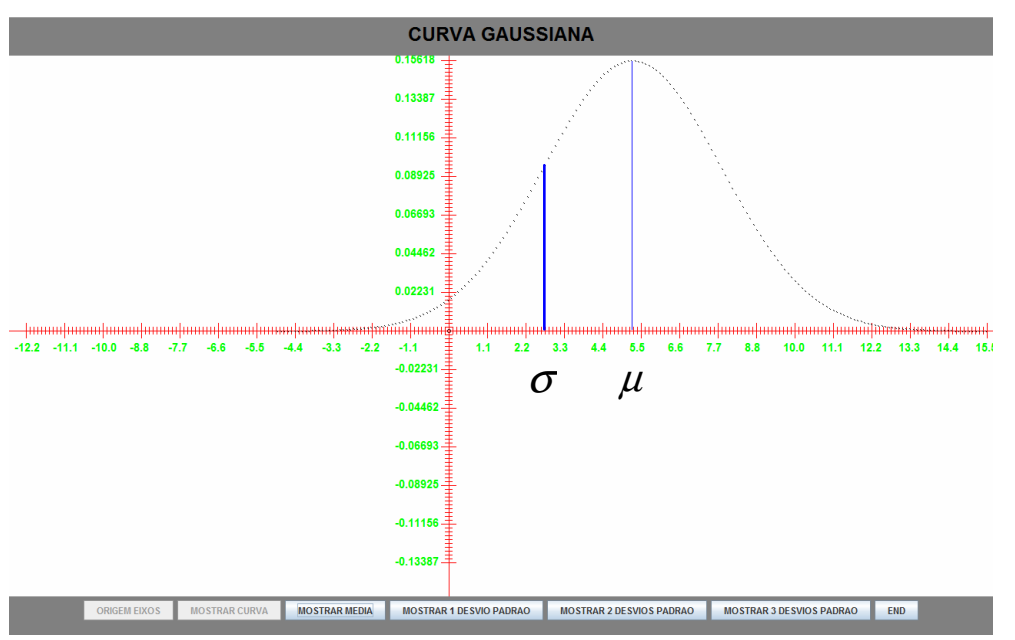


Sejam os conjuntos $\mathrm{X}_{1}, \mathrm{X}_{2}$ e $\mathrm{X}_{3}$ contendo os valores mostrados na tabela 2.

\begin{tabular}{|l|c|c|c|c|c|c|c|c|c|c|c|c|c|c|c|}
\hline $\mathbf{X}_{1}$ & $-\mathbf{4}$ & $-\mathbf{9}$ & $\mathbf{- 7}$ & $\mathbf{- 5}$ & $\mathbf{- 9}$ & $\mathbf{- 8}$ & $\mathbf{- 7}$ & $\mathbf{6}$ & $\mathbf{7}$ & $\mathbf{8}$ & $\mathbf{5}$ & $\mathbf{4}$ & $\mathbf{9}$ & $\mathbf{6}$ & $\mathbf{4}$ \\
\hline $\mathrm{X}_{2}$ & -6 & -5 & -7 & -5 & -6 & -8 & -7 & $\mathbf{6}$ & $\mathbf{7}$ & $\mathbf{6}$ & $\mathbf{5}$ & $\mathbf{4}$ & $\mathbf{6}$ & $\mathbf{6}$ & $\mathbf{4}$ \\
\hline $\mathrm{X}_{3}$ & -14 & -19 & -17 & -15 & -19 & -18 & -17 & 14 & 19 & 17 & 15 & 19 & 18 & 17 & 17 \\
\hline
\end{tabular}

Tabela 2 - conjuntos $X_{1}, X_{2}, X_{3}$

Nesta tabela, as médias das curvas, mostradas na figura 3, são todas iguais a zero e os valores dos respectivos desvios padrões são iguais a 7.010, 7.176 e 17.647, os quais estimam a dispersão dos resultados dos conjuntos de medidas, que medem como os dados estão agrupados em torno da média. Como pode ser visto nas figuras, a curva de Gauss tem a forma de um sino, onde o desvio padrão mede o tamanho da abertura da boca, ou seja, quanto maior este valor, maior é o tamanho da boca do sino e logo menor é a altura deste sino.

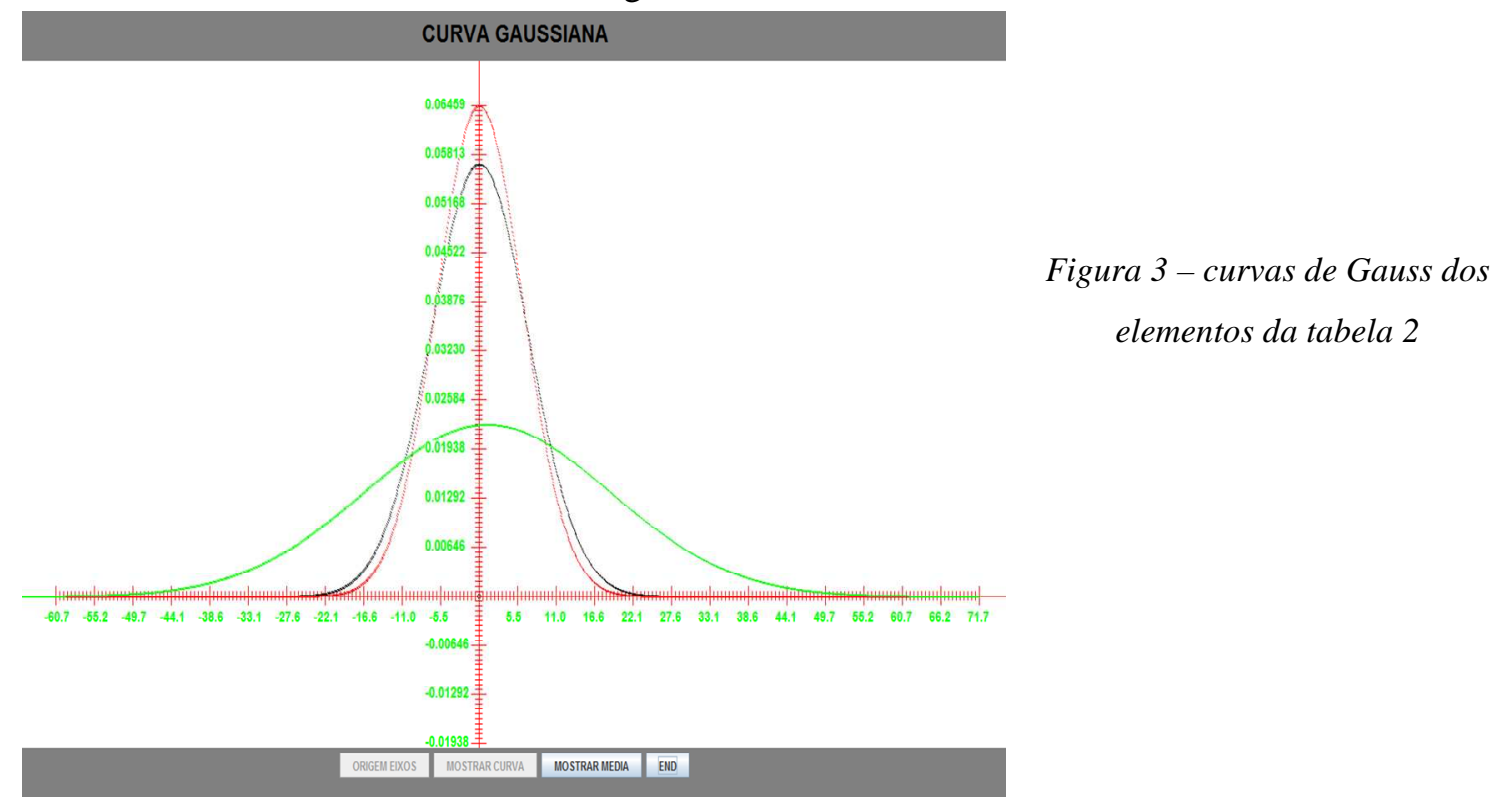

\section{Processamento de Imagens Digitais}

Uma imagem digital tem como representação uma matriz bidimensional contendo números binários codificados de modo a permitir seu armazenamento, transferência, impressão ou reprodução, e seu processamento por meios eletrônicos. No processamento de imagens, a imagem representada pela uma matriz, é constituída dos valores funções da luz incidente sobre os objetos presentes na cena da imagem, ou seja, os pixels ( picture elements ), que apresentam uma correspondência bit-a-bit entre os pontos da imagem e os pontos da imagem reproduzida na tela de um monitor. Eles definem cor, textura, dimensão e posição dos elementos da imagem.

A área de Processamento de Imagens em geral abrange operações que são realizadas sobre imagens e que resultam em imagens [1], [2]. A área de visualização abrange as operações de síntese de imagem, ou seja, a geração de uma visualização do modelo. A área de visão abrange as operações de análise dos objetos definidos pela imagem e a geração de modelos matemáticos desses objetos. No entanto, antes destas operações poderem ser realizadas, uma operação muito importante em processamento de imagens deve ser também realizada: o préprocessamento da imagem. Nesta operação, todo o tratamento da imagem é realizado antes mesmo da imagem ser processada para seu verdadeiro fim. É neste contexto que este trabalho se encaixa, ou seja, operações pontuais realizadas nos pixels da imagem, com o objetivo de tratá-la.

Uma operação pontual importante no processamento da imagem é a digitalização da imagem, ou seja, quando esta é capturada por um equipamento qualquer, como, por exemplo, uma câmera digital, ela deve ser armazenada eletronicamente, e isto é realizado, transformando os sinais da luz incidente na câmera em sinais digitais ( os pixels ). Esta operação gera a 
imagem digital propriamente dita, e é realizada através de outra operação: o histograma da imagem, que consiste na elaboração de um mapa que contabiliza a freqüência de ocorrência dos níveis de cinza na imagem, ou seja, quantas vezes uma determinada cor está presente na imagem.

Durante a operação de captura da imagem, que é sempre dependente da luz incidente, podem ocorrer problemas nesta operação, e por isto após esta operação, são usados os chamados filtros digitais, os quais têm a função de operar uma imagem digital com o objetivo de melhorá-la no sentido de poder ser processada para os fins a que se destina.

\section{Filtragem Espacial}

A freqüência espacial de uma imagem é dada pelas variações de intensidade ( brilho ) por unidade de distancia. Se numa determinada área da imagem há pouca variação de brilho, a frequiência espacial é baixa. De modo inverso, a alta frequiência espacial é revelada pela grande variação de tonalidade por unidade de distancia. Portanto, a freqüência espacial descreve as funções de brilho de uma imagem [4].

Numa imagem digital, os limites entre diferentes materiais, expressos pela tonalidade, podem ser bruscos ou suaves. Os limites com acentuado gradiente são denominados bordas. As bordas são mais freqüentes e nítidas em áreas com alta freqüência espacial, como por exemplo, áreas agrícolas, com alta diversidade de cultivos, limites entre áreas sombreadas e iluminadas, áreas urbanas, malha rodoviária. As cenas homogêneas, tais como vegetação natural, monoculturas, por outro lado, apresentam variações gradativas.

A filtragem espacial de freqüência consiste de operações espaciais que filtram determinadas freqüências no sentido de suavizar, como ocorre nos filtros de passa baixa, ou realçar, como ocorre nos filtros de passa alta. De uma forma geral, filtros digitais tratam com as frequiências das imagens, e o uso dos mesmos, depende da finalidade do aplicativo no qual eles são utilizados.

Um filtro pode ser implementado computacionalmente através de dois processos: convolução e analise de Fourier. A convolução opera no domínio da freqüência espacial da imagem e seu uso é mais comum no processamento de imagens, ao contrario que a analise de Fourier que é constituída de operações matemáticas complexas para separar as diversas freqüências espaciais da imagem.

\section{Convolução}

Chama-se convolução ao processo de filtragem efetuado pelo deslocamento de uma mascara ( filtro ) sobre a imagem, pelo avanço de um pixel por vez. Define-se uma mascara uma janela composta por um numero impar de linhas e colunas. A filtragem compõe-se do calculo do valor do pixel central da mascara na imagem, em função do conjunto de pixels da área coberta pela mascara. O processo é repetido para todos os pixels da imagem, incluindo os da borda, para os quais é criada uma borda falsa ( geralmente com valores iguais a zero ) na imagem.

Como exemplo, seja a imagem mostrada na fig. 4a e a mascara mostrada na fig. 4b. Convoluindo a mascara sobre o trecho marcado na imagem, o novo valor $\mathrm{A}_{3}$ ( que corresponde ao ponto central da mascara ) na imagem será igual a: $A_{22} M_{00}+A_{23} M_{01}+A_{24} M_{02}+A_{32} M_{10}+$ $A_{33} M_{11}+A_{34} M_{12}+A_{42} M_{20}+A_{43} M_{21}+A_{44} M_{22}$.

\begin{tabular}{|c|c|c|c|c|c|c|c|}
\hline $\mathrm{A}_{00}$ & $\mathrm{~A}_{01}$ & $\mathrm{~A}_{02}$ & $\mathrm{~A}_{03}$ & $\mathrm{~A}_{04}$ & $\mathrm{~A}_{05}$ & $\ldots \ldots$ & $\mathrm{A}_{0 \mathrm{n}}$ \\
\hline $\mathrm{A}_{10}$ & $\mathrm{~A}_{11}$ & $\mathrm{~A}_{12}$ & $\mathrm{~A}_{13}$ & $\mathrm{~A}_{14}$ & $\mathrm{~A}_{15}$ & $\ldots \ldots$ & $\mathrm{A}_{1 \mathrm{n}}$ \\
\hline $\mathrm{A}_{20}$ & $\mathrm{~A}_{21}$ & $\mathrm{~A}_{22}$ & $\mathrm{~A}_{23}$ & $\mathrm{~A}_{24}$ & $\mathrm{~A}_{25}$ & $\ldots \ldots$ & $\ldots \ldots$. \\
\hline$\ldots \ldots$ & $\mathrm{A}_{31}$ & $\mathrm{~A}_{32}$ & $\mathrm{~A}_{33}$ & $\mathrm{~A}_{34}$ & $\mathrm{~A}_{35}$ & $\ldots \ldots$ & $\ldots \ldots$. \\
\hline$\ldots \ldots$ & $\mathrm{A}_{41}$ & $\mathrm{~A}_{42}$ & $\mathrm{~A}_{43}$ & $\mathrm{~A}_{44}$ & $\mathrm{~A}_{45}$ & $\ldots \ldots$ & $\ldots \ldots$. \\
\hline$\ldots \ldots$ & $\mathrm{A}_{51}$ & $\mathrm{~A}_{52}$ & $\mathrm{~A}_{53}$ & $\mathrm{~A}_{54}$ & $\mathrm{~A}_{55}$ & $\ldots \ldots$ & $\ldots \ldots$. \\
\hline$\ldots \ldots$ & $\ldots \ldots$. & $\ldots \ldots$. & $\ldots \ldots$. & $\ldots \ldots$ & $\ldots \ldots$ & $\ldots \ldots$ & $\ldots \ldots$. \\
\hline $\mathrm{A}_{\mathrm{n} 0}$ & $\mathrm{~A}_{\mathrm{n} 1}$ & $\mathrm{~A}_{\mathrm{n} 2}$ & $\ldots \ldots$ & $\ldots \ldots$ & $\ldots \ldots$ & $\ldots \ldots$. & $\mathrm{A}_{\mathrm{nn}}$ \\
\hline
\end{tabular}

Figura $4 a-$ Imagem

\begin{tabular}{|l|l|l|}
\hline$M_{00}$ & $M_{01}$ & $M_{02}$ \\
\hline$M_{10}$ & $M_{11}$ & $M_{12}$ \\
\hline$M_{20}$ & $M_{21}$ & $M_{22}$ \\
\hline
\end{tabular}

Figura $4 b$ - mascara 


\section{Filtro Gaussiano}

Um filtro gaussiano é utilizado para borrar ou desfocar a imagem na qual ele é aplicado com o objetivo de reduzir os ruídos presentes na imagem [6] e [7]. O resultado desta operação é a suavização da imagem, relembrando a visualização da mesma através de uma tela translúcida ou como se tivesse sendo vista através de uma lente fora de foco. A suavização gaussiana é largamente utilizada no estágio de pré-processamento da imagem a fim de enaltecer a estrutura da imagem em diferentes escalas.

Matematicamente, a aplicação do filtro gaussiano é realizada da mesma forma que a convolução da imagem com uma função gaussiana, como um filtro passa baixa, ou como um filtro de média simples.

Esta função gaussiana expressa a distribuição normal em estatística e é mostrada na equação 1, utilizada para definir a curva gaussiana de uma dimensão. Como uma imagem é definida em duas dimensões, a função gaussiana utilizada no filtro gaussiano aplicado a ela, também deve ser definido em duas dimensões, ou seja, uma dimensão em $\mathrm{X}$ e outra dimensão em Y. Isto é obtido multiplicando-se a equação 1 definida em $X$ pela equação 1 definida em $Y$, obtendo-se assim, a equação 6.

$$
G(x, y)=G(x) \cdot G(y)^{t}=\frac{1}{2 \pi \sigma^{2}} e^{-\frac{x^{2}+y^{2}}{2 \sigma^{2}}}
$$

Esta distribuição tem $\mathrm{o}$ formato mostrado na figura 5 , onde $\mathrm{x}$ é a distancia da origem no eixo horizontal e y é a distancia da origem no eixo vertical, e $\sigma$ é o desvio padrão da distribuição gaussiana dos pixels que compõem a imagem.

Figura 5

Curva gaussiana bidimensional

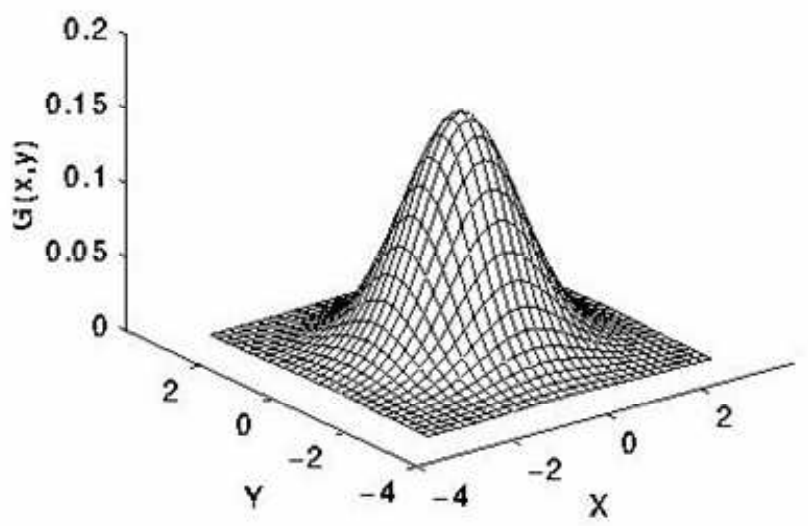

Quando aplicada em duas dimensões, esta formula produz uma superfície cujos contornos são círculos concêntricos, onde o desvio padrão define a abertura do sino formado pela curva.

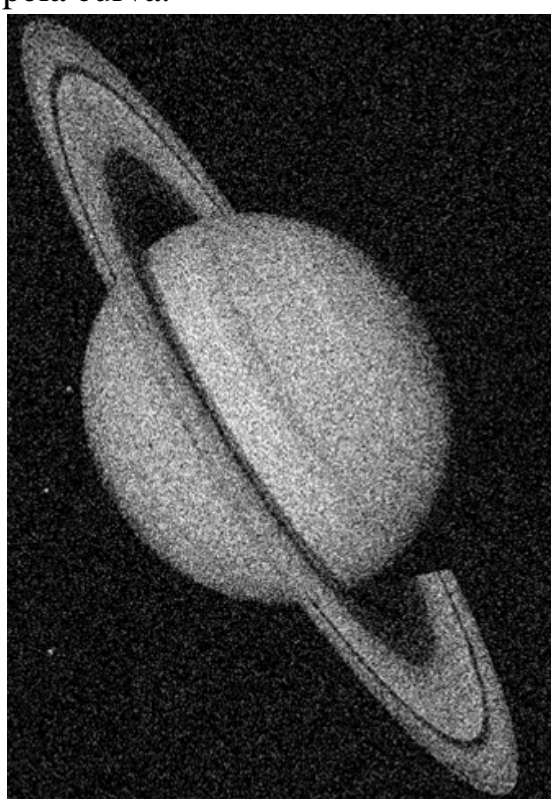

Figura 6

Imagem Original

Figura 7

Filtro Gaussiano

Desvio padrão $=1$

Mascara $3 \times 3$

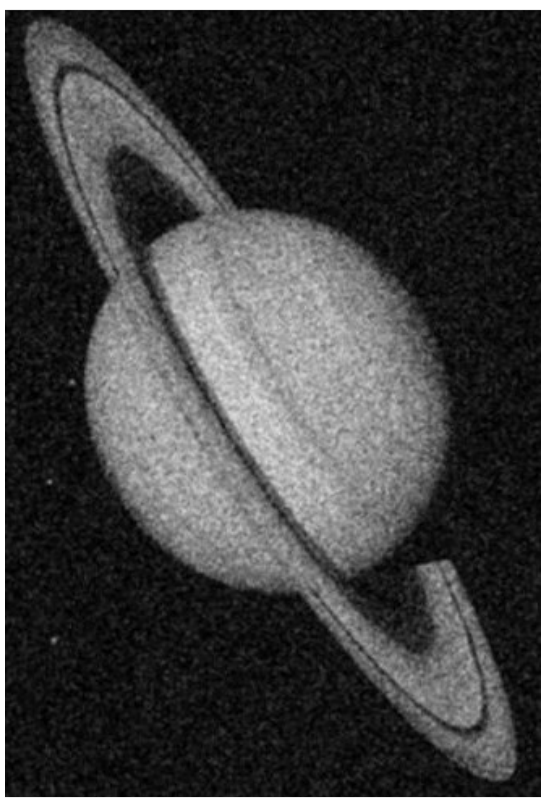


A filtragem gaussiana é comumente aplicada quando se deseja reduzir ruídos na imagem, como mostrado nas figuras 6 e 7. Na figura 6 é mostrado a imagem de Plutão e na figura 7 é mostrada a imagem de Plutão suavizada por um filtro Gaussiano de dimensões 3x3 e com desvio padrão igual a 1.

O filtro gaussiano utilizado na filtragem da imagem é obtido através da geração de uma matriz gaussiana que será utilizada como mascara na convolução da imagem. Esta matriz é gerada utilizando-se a equação 6, atribuindo os valores de x, y e $\sigma$. Desta forma, para uma matriz de dimensão 5, com desvio padrão igual a 1 e ambos, $x$ e y variando entre -2 e 2 , tem-se a estrutura matricial constituída dos valores mostrados na tabela 3.

\begin{tabular}{|l|l|l|l|l|}
\hline 0.00078631 & 0.00655952 & 0.01330347 & 0.00655952 & 0.00078631 \\
\hline 0.00655952 & 0.05472049 & 0.11097944 & 0.05472049 & 0.00655952 \\
\hline 0.01330347 & 0.11097944 & 0.22507904 & 0.11097944 & 0.01330347 \\
\hline 0.00655952 & 0.05472049 & 0.11097944 & 0.05472049 & 0.00655952 \\
\hline 0.00078631 & 0.00655952 & 0.01330347 & 0.00655952 & 0.00078631 \\
\hline \multicolumn{7}{|c|}{ Tabela 3 - exemplo de um filtro gaussiano } \\
\hline
\end{tabular}

Pode-se observar na tabela 3, que o maior valor ( o pico da curva ) encontra-se no centro da tabela, e os seus vizinhos vão diminuindo de valor circularmente, dando a idéia do sino mostrado na figura 5, exatamente como se deseja no comportamento gaussiano. Dependendo do uso do aplicativo deste filtro, este pode ser ainda normalizado, ou seja, ter todos seus valores divididos pela média de todos os valores da matriz. Assim sendo, não é possível obter-se os valores que compõem esta matriz, como por exemplo, o desvio padrão, a partir da própria matriz, ou seja, fornecendo os valores dos parâmetros da equação 6 chega-se à tabela 3, mas o inverso não é verdadeiro. Utilizando o principio da separabilidade deste filtro na equação 1 com os mesmos valores de $\mathrm{x}$ e $\sigma$ e novamente com os mesmos valores de y e $\sigma$, obtém-se respectivamente, os vetores $\mathrm{G}(\mathrm{x})=\mathrm{G}(\mathrm{y})=0.02804|0.23392| 0.47442|0.23392|$ 0.02804 , que multiplicados entre si, conforme a equação $G(x, y)=G(x) G(y)^{t}$, resultam na matriz mostrado na tabela 3 .

\section{Aplicativo Computacional}

As figuras 6 e 7 mostram os resultados obtidos por um aplicativo computacional especialmente desenvolvido para este fim. Este aplicativo, escrito em linguagem Java padrão ANSI [3], tem como entrada de dados, o nome do arquivo onde se encontra a imagem a ser utilizada na entrada de dados do aplicativo, ou seja, a imagem a ser transformada, ou neste caso, filtrada. Na verdade, o aplicativo em questão, denominado LAB IMAGE, mostrado na figura 8 , tem várias outras funções praticas relacionadas ao processamento de imagem, tais como, filtragem da imagem utilizando outros tipos de filtros, rotação, armazenamento, entropia e ampliação da imagem processada.

Como se está interessado apenas na filtragem da imagem utilizando-se um filtro gaussiano, o software permite ao usuário escolher o tamanho da máscara, que pode ser de $3 \times 3$ ( padrão ) até $11 \times 11$, dependendo da experiência a ser elaborada, lembrando que quanto maior for o tamanho da mascara, mais operações são realizadas na convolução da mascara com a imagem. O usuário pode escolher também o valor do desvio padrão, que inicialmente é definido como sendo igual a 1 , mas pode ter seu valor alterado.

\section{Conclusões}

Foram mostrados no texto, os conceitos básicos sobre a Curva de Gauss, utilizada para o calculo do desvio padrão dos valores envolvidos numa amostra e sua extensão na aplicação bidimensional no tratamento de imagens computacionais, utilizados como filtros passa baixa. A fim de demonstrar os conceitos mostrados, foi desenvolvido um aplicativo computacional que tem como entrada uma dada imagem e como saída esta imagem processada com uma determinada função especificada no software e escolhida pelo usuário. No caso deste trabalho, está se interessado apenas pela opção de filtragem da imagem utilizando-se um filtro gaussiano, 
com escolha do tamanho das dimensões da mascara do filtro e também do valor do desvio padrão dos valores da imagem.

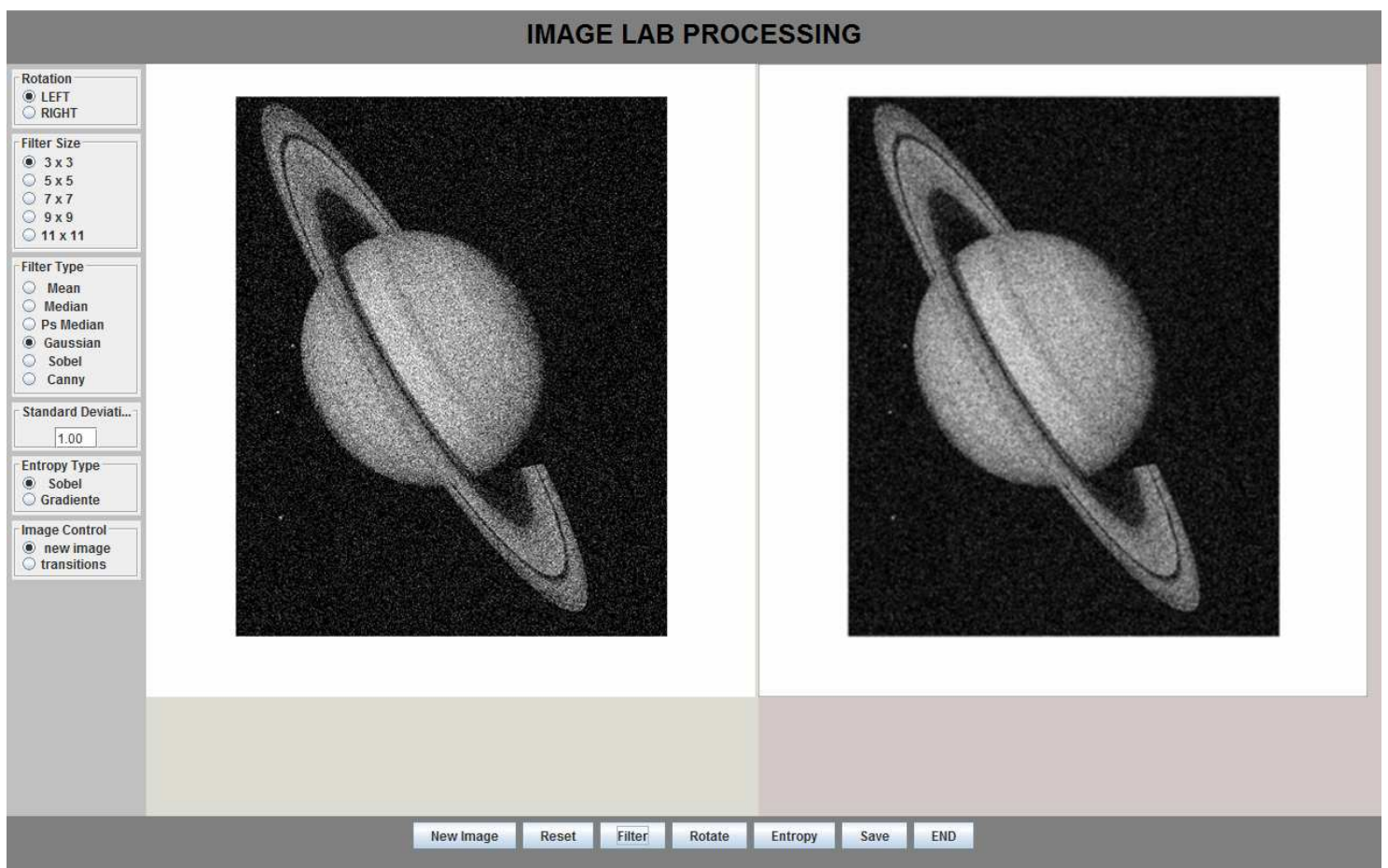

Figura 8 - programa IMAGE LAB

É um aplicativo ideal para o treinamento dos alunos em programas multidisciplinares, tais como, matemática, computação gráfica e processamento digital de imagens. Além disto, como foi desenvolvido pelos autores, tem-se a propriedade do programa fonte, podendo-se altera-lo para incluir, remover ou modificar funções de usuários dentro do aplicativo.

\section{Referências Bibliográficas}

[1] IBGE, Diretoria de Geociências, "Introdução ao Processamento Digital de Imagens", Manuais Técnicos em Geociências, numero 9, 2000.

[2] Rafael C. Gonzalez, Richard E. Woods, "Processamento Digital de Imagens", $3^{\text {a }}$ edição, Pearson, 2011

[3] Douglas A. Lyon, "Image Processing in JAVA", Prentice Hall, 1999

[4] Luiz Pasquali, http://www.psi-ambiental.net/pdf/PasqCap03.pdf , "A Curva Normal", acessado em 10/02/2014

[5] http://pt.wikipedia.org/wiki/Distribuição_normal, "Distribuição Normal", acessado em $10 / 02 / 2014$.

[6] http://en.wikipedia.org/wiki/Gaussian_blur, "Gaussian Blur", acessado em 10/02/2014

[7] http://www.librow.com/articles/article-9, "Gaussian filter, or Gaussian Blur", acessado em $10 / 02 / 2014$ 